Research Paper

\title{
Ebselen Is a Potential Anti-Osteoporosis Agent by Suppressing Receptor Activator of Nuclear Factor Kappa-B Ligand-Induced Osteoclast Differentiation In vitro and Lipopolysaccharide-Induced Inflammatory Bone Destruction In vivo
}

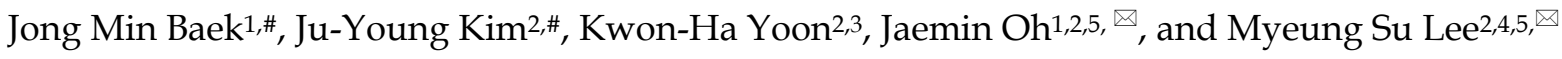 \\ 1. Department of Anatomy, School of Medicine, Wonkwang University, Iksan, Jeonbuk 570-749, Korea \\ 2. Imaging Science-based Lung and Bone Diseases Research Center, Wonkwang University, Iksan, Jeonbuk 570-749, Korea \\ 3. Department of Radiology, School of Medicine, Wonkwang University, Iksan, Jeonbuk 570-749, Korea \\ 4. Division of Rheumatology, Department of Internal Medicine, Wonkwang University, Iksan, Jeonbuk 570-749, Korea \\ 5. Institute for Skeletal Disease, Wonkwang University, Iksan, Jeonbuk 570-749, Korea \\ \# These authors contributed equally to this work.
}

$\triangle$ Corresponding authors: Jaemin $\mathrm{Oh}^{1}$ and Myeung Su Lee'2. ${ }^{1}$ Department of Anatomy, School of Medicine, Wonkwang University, 460 Iksandae-ro, Iksan, Jeonbuk 570-749, Korea. Fax: +82 63852 9115; E-mail address: jmoh@wku.ac.kr (J. Oh). 2Division of Rheumatology, Department of Internal Medicine, School of Medicine, Wonkwang University, 460 Iksandae-ro, Iksan, Jeonbuk 570-749, Korea. Fax: +82 63852 2661; E-mail address: ckhlms@wku.ac.kr (M.S. Lee)

(c) Ivyspring International Publisher. Reproduction is permitted for personal, noncommercial use, provided that the article is in whole, unmodified, and properly cited. See http://ivyspring.com/terms for terms and conditions.

Received: 2015.09.11; Accepted: 2016.01.16; Published: 2016.02.18

\begin{abstract}
Ebselen is a non-toxic seleno-organic drug with anti-inflammatory and antioxidant properties that is currently being examined in clinical trials to prevent and treat various diseases, including atherosclerosis, stroke, and cancer. However, no reports are available for verifying the pharmacological effects of ebselen on major metabolic bone diseases such as osteoporosis. In this study, we observed that ebselen suppressed the formation of tartrate-resistant acid phosphatase (TRAP)-positive multinucleated cells in an osteoblast/osteoclast co-culture by regulating the ratio of receptor activator of nuclear factor kappa-B ligand (RANKL)/osteoprotegerin secreted by osteoblasts. In addition, ebselen treatment in the early stage of osteoclast differentiation inhibited RANKL-dependent osteoclastogenesis by decreasing the phosphorylation of IKB, PI3K, and Akt in early signaling pathways and by subsequently inducing c-Fos and nuclear factor of activated T-cells cl. Further, ebselen induced apoptosis of osteoclasts in the late stage of osteoclast differentiation. In addition, ebselen treatment suppressed filamentous actin ring formation and bone resorption activity of mature osteoclasts. Reflecting these in vitro effects, administration of ebselen recovered bone loss and its $\mu$-CT parameters in lipopolysaccharide-mediated mouse model. Histological analysis confirmed that ebselen prevented trabecular bone matrix degradation and osteoclast formation in the bone tissues. Finally, it was proved that the anti-osteoclastogenic action of ebselen is achieved through targeting N-methyl-D-aspartate (NMDA) receptor. These results indicate that ebselen is a potentially safe drug for treating metabolic bone diseases such as osteoporosis.
\end{abstract}

Key words: Ebselen; Osteoclast; Bone resorption; Osteoporosis

\section{Introduction}

Osteoporosis is a typical aging-related disorder that decreases bone mass and subsequently increases fragility, thus increasing the risk of fractures [1]. Se- nile osteoporosis is mainly attributed to several pathological conditions associated with senescence, such as production of inflammatory cytokines and 
reactive oxygen species (ROS). Previous studies have shown that inflamm-aging induces general age-related osteoporosis by increasing the expression levels of inflammatory cytokines such as tumor necrosis factor-alpha (TNF-a) and interleukin-1alpha (IL-1a) $[2,3]$. These cytokines play a critical role in promoting mature osteoclast generation and their bone resorption activity [4]. In addition, IL-1a positively regulates the expression of receptor activator of nuclear factor kappa-B (RANK) ligand (RANKL) in osteoblasts, thus enhancing osteoclast activity, negatively regulating osteoclast apoptosis, and increasing osteoclast life span $[2,5]$. Increased levels of ROS induce age-dependent decrease in bone mass [6]. Oxidative stress stimuli transmitted by increased intracellular production of ROS act as secondary messengers in RANKL-induced osteoclast signaling pathways [7]. ROS regulates RANKL-dependent signal transducers such as p38 and c-Jun N-terminal kinase (JNK), which play an essential role in the early stage of osteoclastogenesis, during the differentiation of monocytes and macrophages into osteoclasts [8, 9]. Furthermore, generation of oxygen-derived free radicals in the bone environment increases osteoclastic bone resorption in vitro and bone matrix degradation in vivo [10]. Therefore, management of inflammation, ROS, and other aging-related factors is important for treating degenerative bone disorders such as osteoporosis.

Ebselen, also called PZ51, is a synthetic selenium-containing compound with low toxicity. It was first identified as a glutathione peroxidase mimic with antioxidant activity and a potent scavenger of membrane-bound phospholipid hydroperoxides [11-13]. Ebselen exerts various pharmacological effects in different biological processes. Ebselen downregulates TNF-a-induced increased expression of proinflammatory cytokines such as IL-6, IL-8, monocyte chemoattractant protein-1, and cyclooxygenase 2 in glioblastoma cells [14]. In addition, ebselen suppresses hydrogen peroxide-mediated apoptosis of PC12 cells by regulating JNK and activator protein-1 signaling pathways, suggesting its therapeutic potential against ROS-associated ischemic cerebral diseases [15]. Ebselen also induces other pharmacological effects, including anti-invasion effect on pancreatic and renal cancer cell lines, antiviral effect on hepatitis $C$ virus nonstructural protein 3 , and antiatherogenic effect on diabetic macrovascular disease [16-18]. Because of these properties, ebselen is currently being examined in clinical trials for managing various diseases, especially those associated with aging, including stroke, atherosclerosis, reperfusion injury, bipolar disorder, and cancer $[13,19]$. However, no reports are available to show the relationship between ebselen and bone diseases such as osteoporosis.

Because ebselen (a part of the National Institutes of Health Clinical Collection) is a bioavailable drug that is clinically safe without a proven use, we performed the present study to determine the potential of ebselen for treating osteoporosis [19]. We investigated the effects of ebselen on RANKL-induced differentiation of osteoclasts and their functions and the underlying molecular mechanisms. Furthermore, we determined the effects of ebselen on lipopolysaccharide (LPS)-induced bone erosion in vivo.

\section{Materials and Methods}

\section{Reagents and antibodies}

Ebselen (2-phenyl-1,2-benzisoselenazol-3[2H]one) was purchased from Cayman Chemical (Ann Arbor, MI, USA) with high purity (more than 99\%) and was dissolved in dimethyl sulfoxide. N-Methyl-D-aspartic acid (NMDA) receptor agonist, NMDA was purchased from Sigma (St. Louis, MO, USA) with high purity (more than 98\%). Recombinant soluble human macrophage colony-stimulating factor (M-CSF), RANKL, and IL-1 $\alpha$ were obtained from PeproTech EC Ltd. (London, UK). A monoclonal antibody against $\beta$-actin was obtained from Sigma (St. Louis, MO, USA). Antibodies against phosphorylated (phospho)-p38, p38, phospho-extracellular signal-regulated protein kinase (ERK), ERK, phospho-JNK, JNK, phospho-IKB, phospho-phosphoinositide 3-kinase (PI3K), PI3K, phospho-Akt, Akt, caspase-3, and caspase-9 were purchased from Cell Signaling Technology Inc. (Beverly, MA, USA). Antibodies against c-Fos, nuclear factor of activated T cells c 1 (NFATc1), and IkB were purchased from Santa Cruz Biotechnology (Santa Cruz, CA, USA). Fetal bovine serum (FBS), $\alpha$-minimum essential medium ( $\alpha$-MEM), penicillin, and streptomycin were purchased from Gibco BRL (Grand Island, NY, USA). All other chemicals were of analytical grade or complied with the standards required for cell culture experiments.

\section{Experimental animals}

Five-week-old male ICR mice were purchased from Samtako Co. Ltd. (Osan, Korea) and were maintained at $22^{\circ} \mathrm{C}-24^{\circ} \mathrm{C}$ and $55 \%-60 \%$ humidity in a room with a 12/12-h light/dark cycle. All experiments were conducted according to the guidelines of the Institutional Animal Care and Use Committee of Wonkwang University (WKU15-111).

\section{Isolation of bone marrow cells and pretreat- ment with ebselen}

Bone marrow cells (BMCs) were isolated from 5 -week-old male ICR mice by flushing their tibias and 
femurs with $\alpha$-MEM supplemented with $10 \%$ FBS, penicillin $(100 \mathrm{U} / \mathrm{mL})$, and streptomycin (100 $\mu \mathrm{g} / \mathrm{mL}$ ). Bone marrow macrophages (BMMs) were obtained by culturing BMCs in $\alpha$-MEM supplemented with $10 \% \mathrm{FBS}$ and M-CSF $(10 \mathrm{ng} / \mathrm{mL})$ at $37^{\circ} \mathrm{C}$ in $5 \%$ $\mathrm{CO}_{2}$ for 1 day. Non-adherent cells were transferred to $10-\mathrm{cm}$ Petri dishes and were further cultured in the presence of M-CSF $(30 \mathrm{ng} / \mathrm{mL})$ for 3 days. Non-adherent cells were removed, and adherent cells were used as BMMs. To generate osteoclasts from BMMs, we cultured BMMs in 48-well plates at $37^{\circ} \mathrm{C} / 5 \% \mathrm{CO}_{2}$ for 4 days in the condition of M-CSF ( 30 $\mathrm{ng} / \mathrm{mL})$ and RANKL $(100 \mathrm{ng} / \mathrm{mL})$ pretreated with ebselen. The cells were then stained with tartrate-resistant acid phosphatase (TRAP) solution as described previously [20].

\section{F-actin ring staining}

BMMs were cultured in 48-well plates in the presence of M-CSF (30 ng/mL) and RANKL (100 $\mathrm{ng} / \mathrm{mL}$ ) for 2 days and were then treated with ebselen for 2 days. The cells were then stained with rhodamine-conjugated phalloidin for filamentous actin (F-actin) ring labeling, as described previously [21]. The change of F-actin ring on mature osteoclasts induced by ebselen was quantified by calculating the ratio of actin ring positive (AR+) osteoclasts versus actin ring negative (AR-) osteoclasts. The osteoclasts with normal F-actin rings were considered as AR+ osteoclasts and osteoclasts without or disrupted F-actin rings were considered as AR- osteoclasts. It was defined as disrupted actin rings if less than half of them showed typical morphology of F-actin rings [22].

\section{Detection of apoptotic body formation}

BMMs were pre-treated with ebselen in the presence of M-CSF (30 ng/mL) and RANKL (100 $\mathrm{ng} / \mathrm{mL}$ ) for 2 days and BMMs were post-treated with ebselen for 2 days of culture in the presence of M-CSF $(30 \mathrm{ng} / \mathrm{mL})$ and RANKL $(100 \mathrm{ng} / \mathrm{mL})$. The cells were then stained with DAPI solution as described previously [21].

\section{TUNEL assay}

BMMs were pretreated with ebselen in the presence of M-CSF (30 ng/mL) and RANKL (100 $\mathrm{ng} / \mathrm{mL}$ ) for 2 days and BMMs were posttreated with ebselen for 2 days of culture in the presence of M-CSF (30 ng/mL) and RANKL (100 ng/mL). After, to detect the formation of apoptotic osteoclasts, we performed TUNEL (TdT-mediated dUTP-biotin nick end-labeling) assay by using ApopTag ${ }^{\circledR}$ Fluorescein In Situ Apoptosis Detection Kit (Millipore, Merck KgaA, Darmstadt, Germany) according to the manu- facturer's protocol.

\section{Co-culture of BMCs and calvaria primary os- teoblasts}

Primary osteoblasts $\left(2.5 \times 10^{4}\right.$ cells/well $)$ and BMCs $\left(2.5 \times 10^{5}\right.$ cells/well $)$ were co-cultured in 48 -well plates in the presence of $10^{-8} \mathrm{M} 1,25(\mathrm{OH})_{2} \mathrm{D}_{3}$ (Vit $\left.\mathrm{D}_{3}\right)$ (Sigma) and $10^{-6} \mathrm{M}$ prostaglandin $\mathrm{E}_{2}\left(\mathrm{PGE}_{2}\right.$; Sigma) or IL-1 $\alpha$ (10 ng/mL) for 7 days. The co-cultured cells were then stained with TRAP solution.

\section{Cytotoxicity assay, western blotting, and re- al-time reverse transcription polymerase chain reaction (real-time $R T-P C R$ )}

XTT assay, western blotting, and real-time RT-PCR were performed as described previously [20]. Primer sets used are shown in Table 1.

Table 1. Primer sequences used for real-time PCR analysis

\begin{tabular}{lll}
\hline Gene name & & Primer sequence $\left(5^{\prime} \rightarrow 3^{\prime}\right)$ \\
\hline GAPDH & Forward & TCA AGA AGG TGG TGA AGC AG \\
& Reverse & AGT GGG AGT TGC TGT TGA AGT \\
DC-STAMP & Forward & TCC TCC ATG AAC AAA CAG TTC CA \\
& Reverse & AGA CGT GGT TTA GGA ATG CAG CTC \\
OC-STAMP & Forward & ATG AGG ACC ATC AGG GCA GCC ACG \\
& Reverse & GGA GAA GCT GGG TCA GTA GTT CGT \\
Cathepsin K & Forward & CCA GTG GGA GCT ATG GAA GA \\
& Reverse & CTC CAG GTT ATG GGC AGA GA \\
CTR & Forward & TCC AAC AAG GTG CTT GGG AA \\
& Reverse & CTT GAA CTG CGT CCA CTG GC \\
Atp6vOd2 & Forward & GAC CCT GTG GCA CTT TTT GT \\
& Reverse & GTG TTT GAG CTT GGG GAG AA \\
\hline
\end{tabular}

\section{Bone resorption assay}

Primary osteoblasts $\left(1 \times 10^{6}\right.$ cells $)$ and BMCs $\left(1 \times 10^{7}\right.$ cells $)$ were cultured in collagen gel-coated culture dishes in the presence of $10^{-8} \mathrm{M}$ Vit $\mathrm{D}_{3}$ and $10^{-6} \mathrm{M} \mathrm{PGE}_{2}$ for 7 days. Co-cultured osteoclasts were detached using $0.1 \%$ collagenase at $37^{\circ} \mathrm{C}$ for $10 \mathrm{~min}$ and were re-seeded in 48-well plates, hydroxyapatite-coated plates (Corning, NY, USA), or dentin slices with or without ebselen. After $6 \mathrm{~h}$, the cells re-seeded in 48-well plates were stained with TRAP solution. The cells re-seeded in hydroxyapatite-coated plates or dentin slices were removed after $24 \mathrm{~h}$, and total number of resorption pits were determined under a microscope and were quantified using Image-Pro Plus version 4.0 (Media Cybernetics, Silver Spring, MD, USA).

\section{Mouse model of LPS-induced bone loss, mi- cro-computed tomography, and histological analysis}

ICR mice were divided into 4 experimental groups comprising 5 mice each: phosphate-buffered 
saline (PBS)-treated (control) group, ebselen only-treated (ebselen) group, LPS only-treated (LPS) group, and LPS- and ebselen-treated (LPS + ebselen) group. Ebselen $(10 \mathrm{mg} / \mathrm{kg})$ or PBS was administered orally every 8 days, and LPS was injected intraperitoneally on days 1 and 4 . The mice were sacrificed after 8 days. Next, micro-computed tomography $(\mu-\mathrm{CT})$ data containing 3D images and bone parameters and histological data were acquired as described previously [20].

\section{Statistical analysis}

All experiments were conducted at least 3 times and data are expressed as mean \pm standard deviation (SD). All statistical analyses were performed using Statistical Package for the Social Sciences Software
A
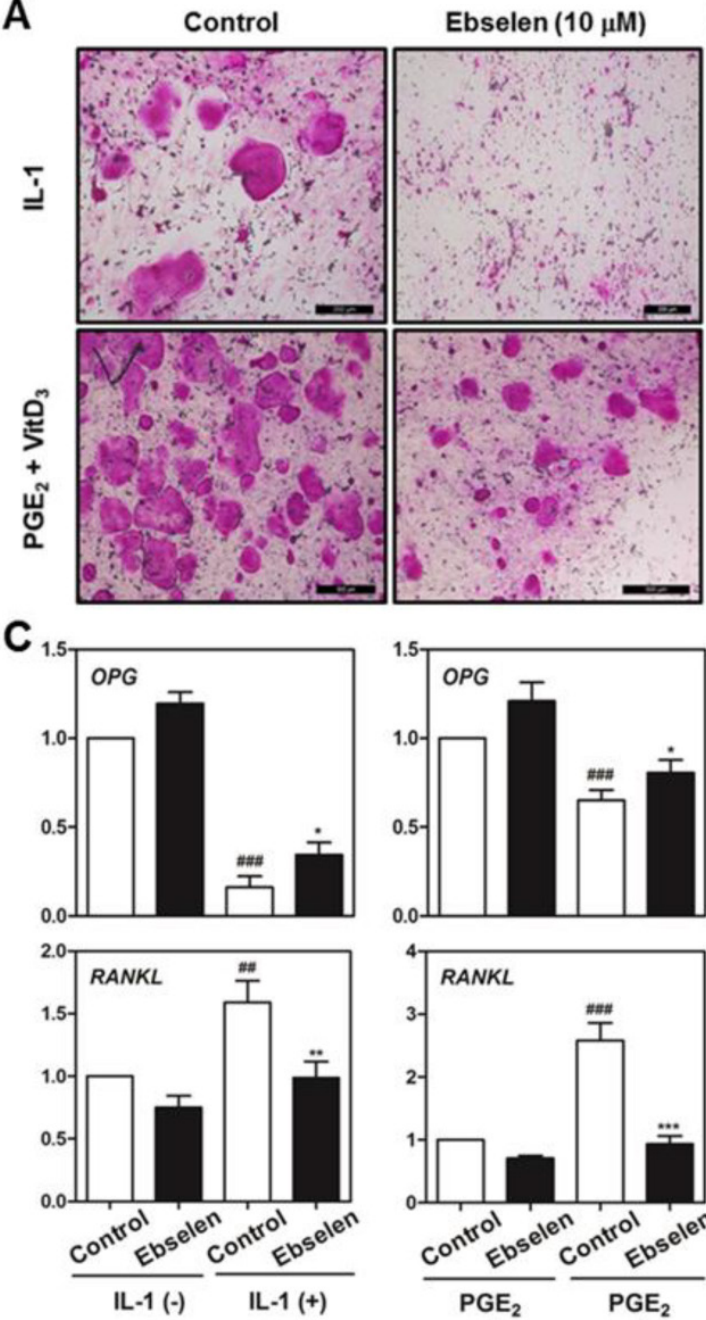

B
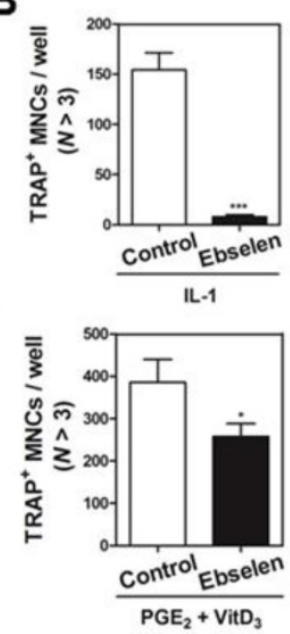

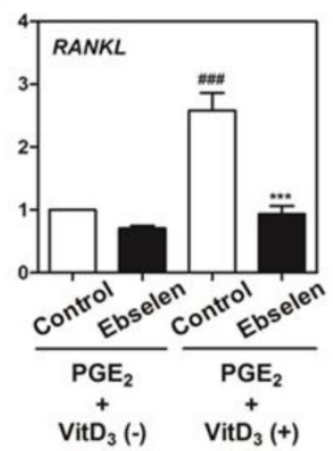

Fig. 1. Ebselen inhibits osteoclast differentiation in the co-culture of BMCs and osteoblasts. (A) Calvaria-derived primary osteoblasts and BMCs were co-cultured for 7 days in a medium containing $\mathrm{IL}-1 \alpha(10 \mathrm{ng} / \mathrm{mL})$ or $\mathrm{VitD}_{3}\left(10^{-8} \mathrm{M}\right)$ and $\mathrm{PGE}_{2}\left(10^{-6} \mathrm{M}\right)$ in the presence or absence of ebselen. (B) The number of TRAP-positive MNCs (nuclei $>3$ ) was determined in these cultures; $n=3,{ }^{* * * * *} P<0.001,{ }^{*} P<$ 0.05 . (C) Primary osteoblasts were pretreated with or without ebselen $(10 \mu \mathrm{M})$ and were cultured in the same conditioned medium for 1 day. Real-time RT-PCR was performed to determine the mRNA expression of genes encoding OPG and RANKL; $n=3$, **** $P<0.001,{ }^{* * *} P<0.01$ and ${ }^{*} P<0.05$ versus control group (IL- $1 \alpha(+)$ or $\mathrm{VitD}_{3}$ and $\left.\mathrm{PGE}_{2}(+)\right)$; $\# P<0.001$ and $\# P<0.05$ versus control group (IL-1 $\alpha$ $(-)$ or VitD ${ }_{3}$ and $\left.\mathrm{PGE}_{2}(-)\right)$.
(SPSS; Korean version 14.0). Student's t-test was used to compare parameters between 2 groups while analysis of variance followed by Tukey post-hoc test was used to compare parameters among 3 groups. $P<0.05$ was considered statistically significant.

\section{Results}

\section{Ebselen suppresses osteoclastogenesis in the co-culture of BMCs and osteoblasts by regu- lating RANKL and osteoprotegerin expression}

We first examined whether ebselen was involved in the formation of TRAP-positive multinucleated cells (MNCs) in the co-culture of BMCs and primary osteoblasts. Ebselen significantly inhibited IL- $1 \alpha$-induced or Vit $\mathrm{D}_{3}$ - and $\mathrm{PGE}_{2}$-induced formation of TRAP-positive MNCs in the co-culture system (Fig. 1A). Ebselen decreased the number of IL-1 $\alpha$-induced or Vit $\mathrm{D}_{3^{-}}$and PGE2-induced TRAP-positive osteoclasts in a dose-dependent manner (Fig. 1B). Further, ebselen decreased mRNA levels of RANKL and increased levels of osteoprotegerin (OPG), a decoy receptor of RANKL that is expressed by osteoblasts, induced by IL- $1 \alpha$ or Vit $\mathrm{D}_{3}$ and $\mathrm{PGE}_{2}$ treatment (Fig. 1C). These results strongly indicated that ebselen negatively regulated the formation of osteoclasts in the co-culture system by controlling the expression of OPG and RANKL.

\section{Ebselen treatment in the early dif- ferentiation stage blocks RANKL-induced osteoclast differen- tiation by regulating early signaling pathways and subsequent c-Fos and NFATcl expression}

To determine the effects of ebselen on the early stage of osteoclastogenesis, BMM cultures were treated with ebselen in the presence of M-CSF and RANKL. Ebselen treatment inhibited osteoclast differentiation in a dose-dependent manner (Fig. 2A). The number of TRAP-positive MNCs significantly decreased in BMM cultures treated with ebselen (Fig. 2B). These inhibitory effects of ebselen did not affect cell viability (Fig. $2 \mathrm{C})$. To elucidate the mechanisms underlying the effects of ebselen on the early stage of osteoclastogenesis, we determined the effects of ebselen on RANKL-induced effectors, including IкB, PI3K, Akt, p38, JNK, and ERK, in the early 
stage of osteoclastogenesis and expression of osteoclast master regulators c-Fos and NFATc1. Ebselen suppressed both RANKL-induced increase in the phosphorylation of IKB, PI3K and Akt and expression of c-Fos and NFATc1. These results showed that ebselen was involved in the early stage of osteoclastogenesis via reducing IKB and PI3K/Akt phosphorylation and leading to subsequent decreased expression of c-Fos and NFATc1.

\section{Ebselen treatment in the late differentiation stage inhibits RANKL-induced osteoclast dif- ferentiation by inducing osteoclast apoptosis.}

To investigate the effects of ebselen on the late stage of osteoclastogenesis, we first post-treated eb- selen in RANKL-induced osteoclast differentiation. Ebselen posttreatment decreased the formation of TRAP-positive MNCs in a dose-dependent manner (Figs. 3A, B). Furthermore, the expression of cleaved caspase-3 and 9 which are apoptosis-related cysteine peptidase was triggered at 24 and $48 \mathrm{~h}$ in the treatment of ebselen during RANKL-induced osteoclast differentiation (Fig. 3C). As shown in Fig. 3D and E, ebselen did not induce apoptosis of osteoclast precursors in early stage, while the formation of apoptotic body and TUNEL-positive apoptotic cells was achieved by post-treatment of ebselen in late stage.
A

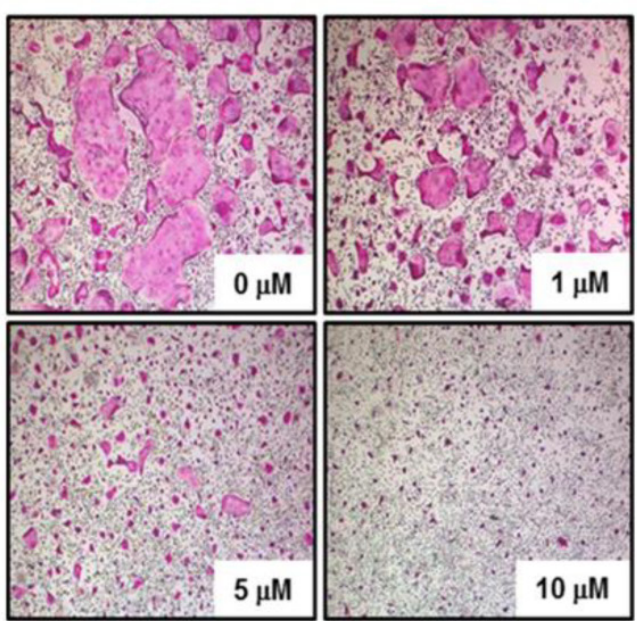

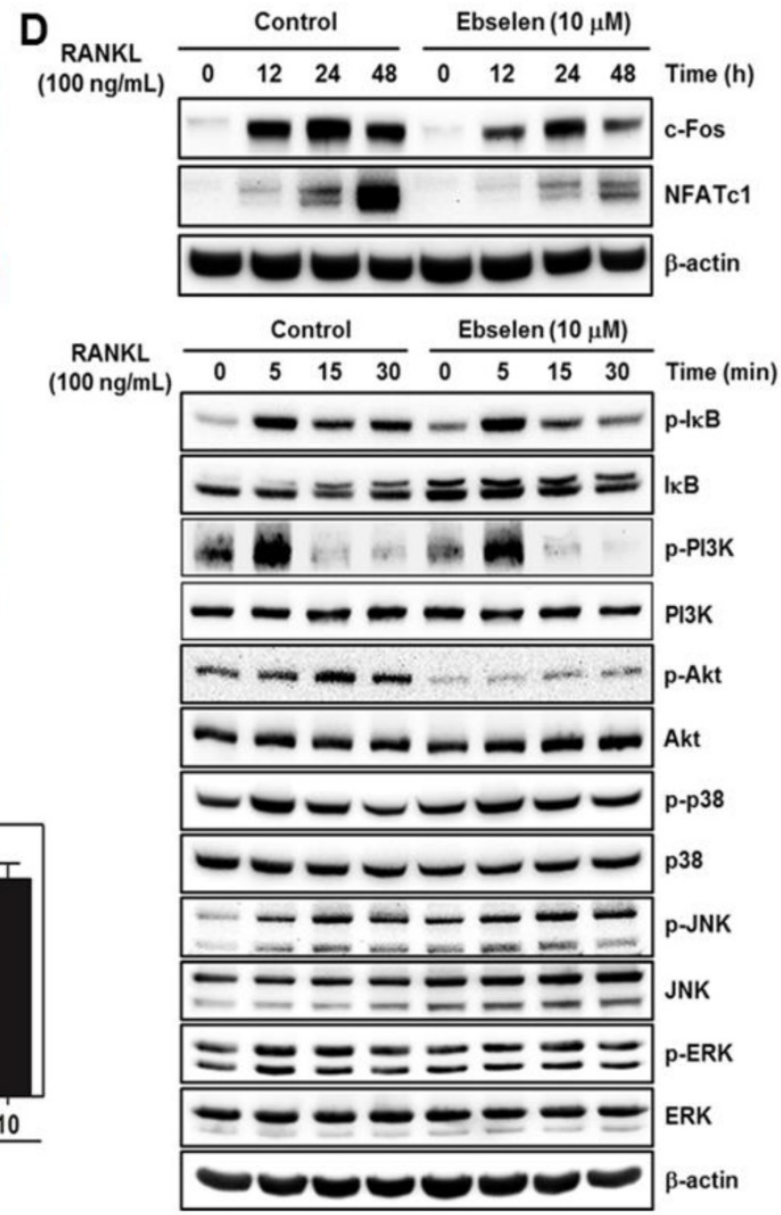

Fig. 2. Ebselen suppresses the early stage of RANKL-induced osteoclastogenesis. (A) BMMs were cultured with $M-C S F(30 \mathrm{ng} / \mathrm{mL})$ and $R A N K L(100 \mathrm{ng} / \mathrm{mL})$ in the $\mathrm{presence}$ or absence of the indicated concentrations of ebselen. The cells were fixed, permeabilized, and stained with TRAP solution. TRAP-positive MNCs were photographed under a light microscope. (B) The number of TRAP-positive MNCs (nuclei $>3$ ) was determined; $n=3,{ }^{* * *} P<0.001$ versus control group. (C) BMMs were seeded in a 96 -well plate and were cultured for 3 days in the presence of M-CSF $(30 \mathrm{ng} / \mathrm{mL})$ and the indicated concentrations of ebselen. Cell viability was analyzed by performing the XTT assay. (D) BMMs were pretreated with or without ebselen for $1 \mathrm{~h}$ in the presence of M-CSF $(30 \mathrm{ng} / \mathrm{mL})$ before RANKL $(100 \mathrm{ng} / \mathrm{mL})$ stimulation at the indicated time points. Whole-cell lysates were analyzed by performing western blotting with the indicated antibodies. $\beta$-actin was used as an internal control.
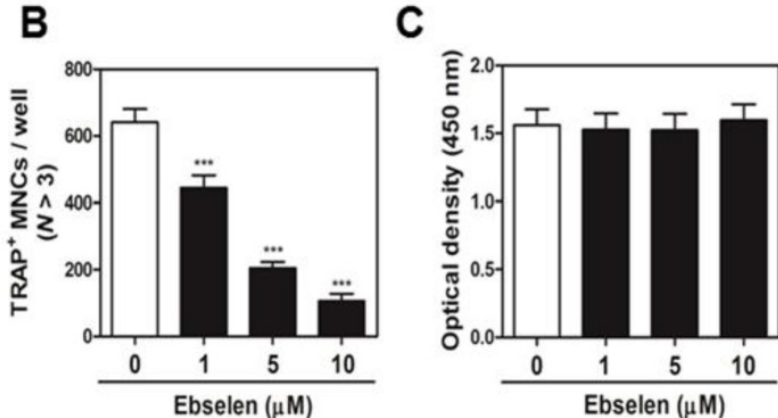
A
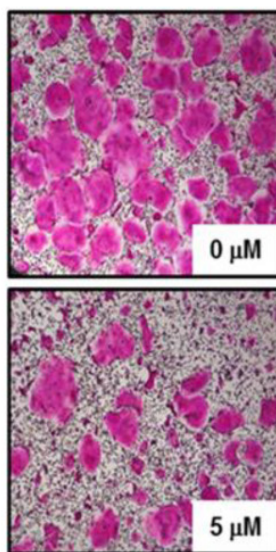

D
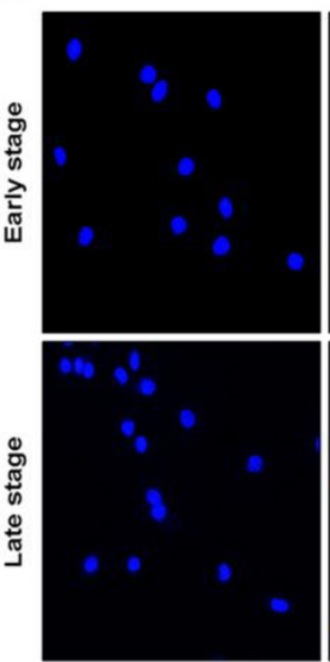

Control

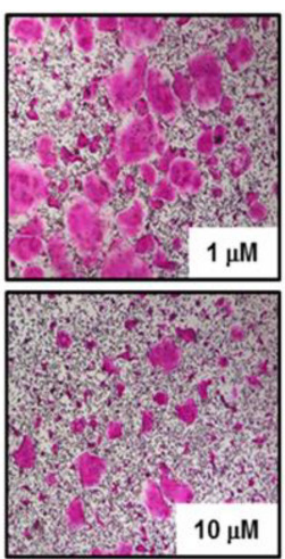

B

E
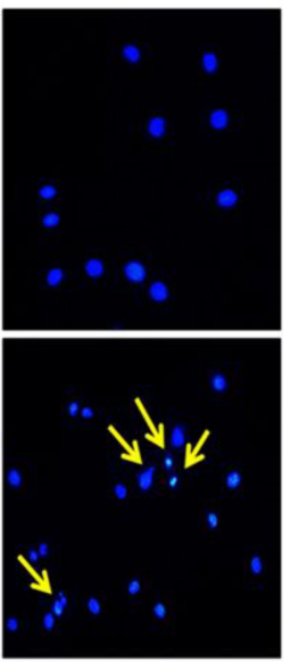

Ebselen

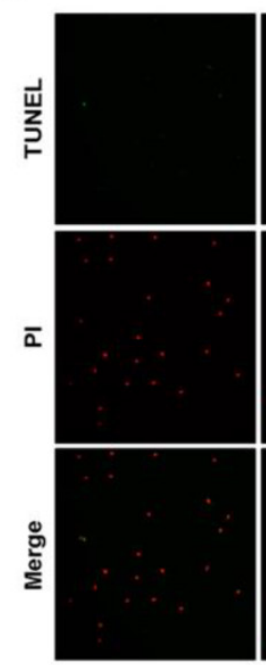

Control

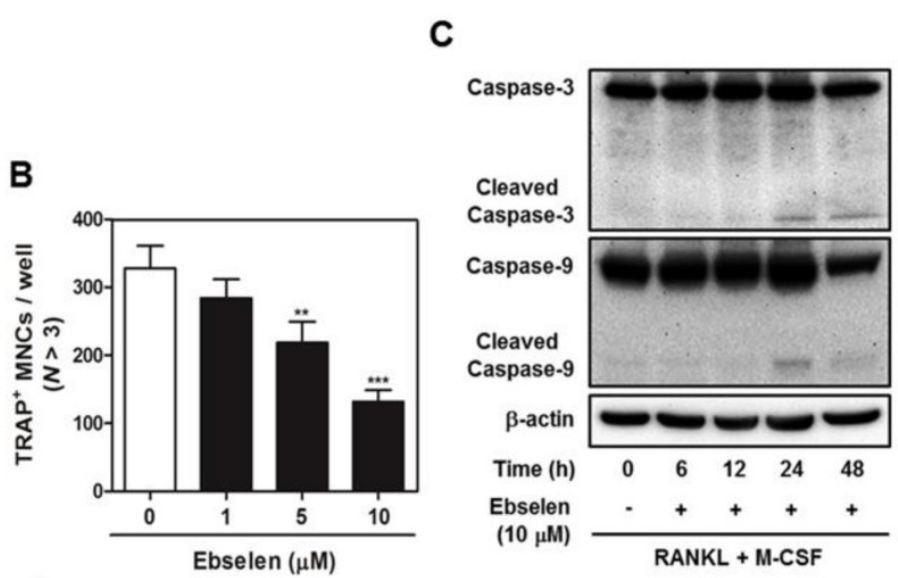

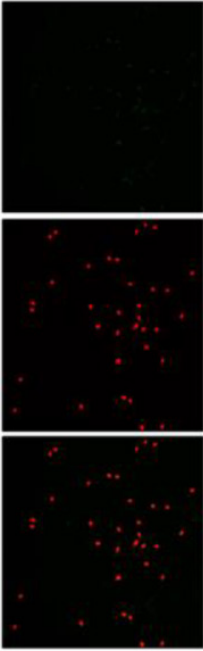

Ebselen Early stage

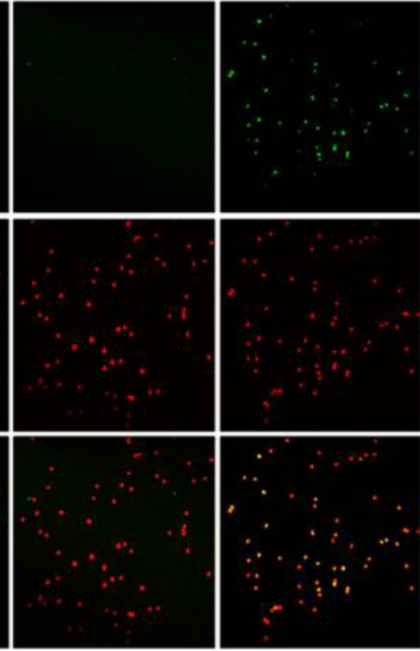

Control Late stage

Fig. 3. Ebselen induces apoptosis in the late stage of RANKL-induced osteoclastogenesis. (A) BMMs were cultured with $M-C S F(30 \mathrm{ng} / \mathrm{mL})$ and $R A N K L(100 \mathrm{ng} / \mathrm{mL})$, after which they were treated with the indicated concentrations of ebselen. The cells were fixed, permeabilized, and stained with TRAP solution. (B) The number of TRAP-positive MNCs (nuclei, $>3$ ) was determined; $n=3$, ${ }^{* * * *} P<0.001$ and ${ }^{* *} P<0.01$ versus control group. (C) BMMs were cultured with $M-C S F(30 \mathrm{ng} / \mathrm{mL})$ and $R A N K L(100 \mathrm{ng} / \mathrm{mL})$ before treatment with ebselen at the indicated time points. Whole-cell lysates were analyzed by performing western blotting with the indicated antibodies. $\beta$-actin was used as an internal control. (D) BMMs were pre- or posttreated with ebselen in the presence of M-CSF (30 ng/mL) and RANKL $(100 \mathrm{ng} / \mathrm{mL})$. The cells were fixed, permeabilized and stained with DAPI and were examined under a confocal laser-scanning microscope. (E) BMMs were cultured as described above and stained with TUNEL and PI and were examined under a confocal laser-scanning microscope.

\section{Ebselen blocks the actin ring formation and bone resorption activity of mature osteoclasts by reducing the expression of osteoclastic marker genes}

Next, we examined whether ebselen blocked the most important features of mature osteoclasts, F-actin structure and bone resorption. Ebselen inhibited the formation of F-actin ring structure, which is formed by the fusion of osteoclasts, in a dose-dependent manner (Figs. 4A, B). In addition, ebselen significantly attenuated the mRNA expression of genes encoding OC- and DC-STAMP, which are required for osteoclast fusion (Fig. 4C). Also, we seeded mature osteoclasts in 48-well plates, hydroxyapatite-coated plates, or dentin slices with or without ebselen to determine the direct effects of ebselen on bone resorption activity without inducing cell death. As shown in (Figs. 4D, E, $\mathrm{F}$, and $\mathrm{G}$ ), while considerable resorption pit formation was observed in the control group, ebselen significantly disrupted the ability of mature osteoclasts to form resorption pits in hydroxyapatite-coated plates or dentin slices with no change in the number TRAP-stained mature osteoclasts. Ebselen also downregulated the expression of several genes encoding transcription factors associated with bone resorption, including cathepsin K, CTR, and Atp6vOd2 (Fig. $4 \mathrm{H}$ ). These results indicated that ebselen directly suppressed main functions of mature osteoclasts by controlling the expression of osteoclast marker genes in vitro. 
A
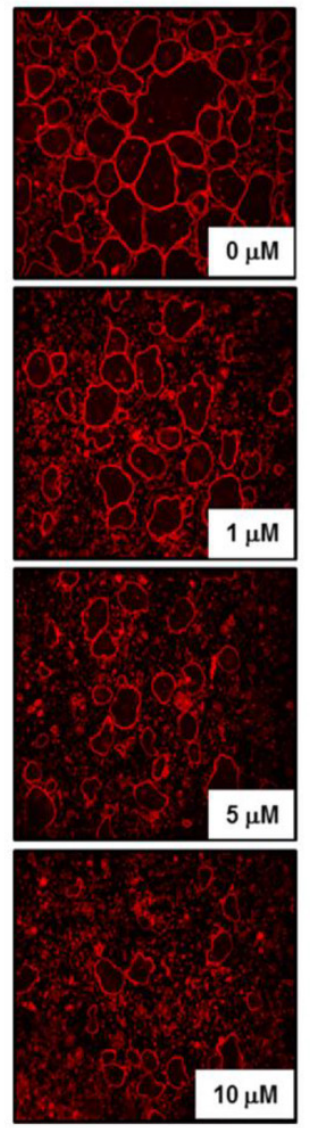

B

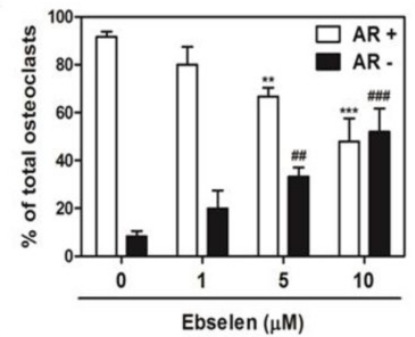

D
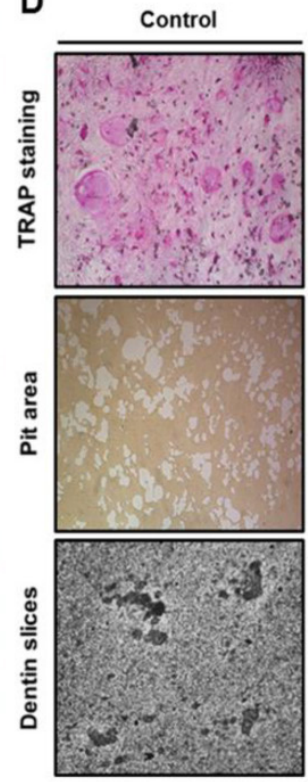

Ebselen $(10 \mu \mathrm{M})$
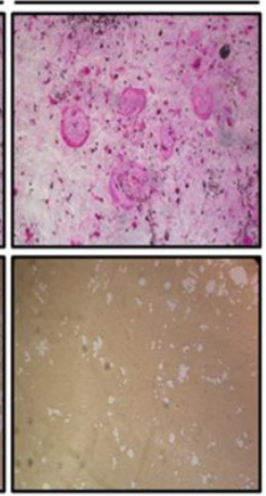

C

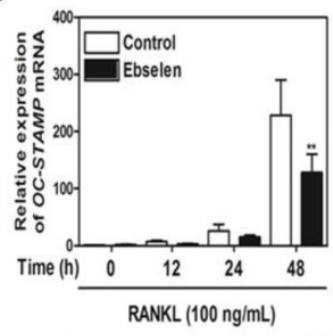

E

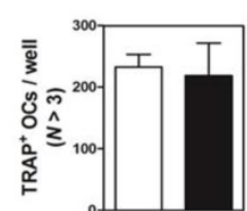

$F$
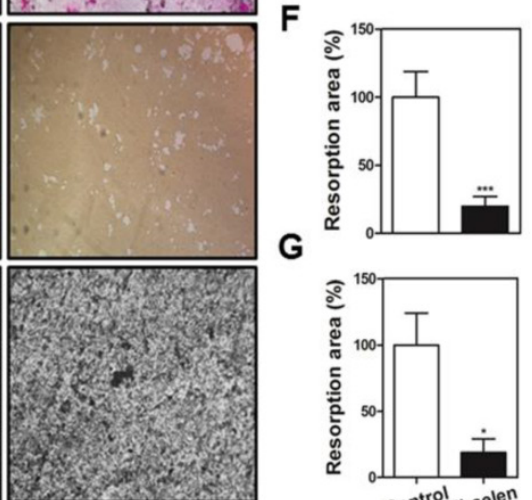

G

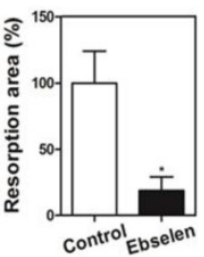

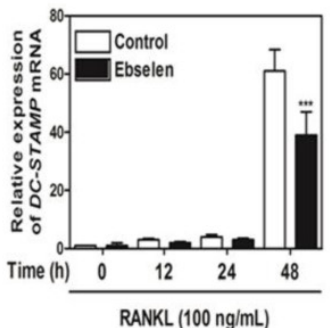

H
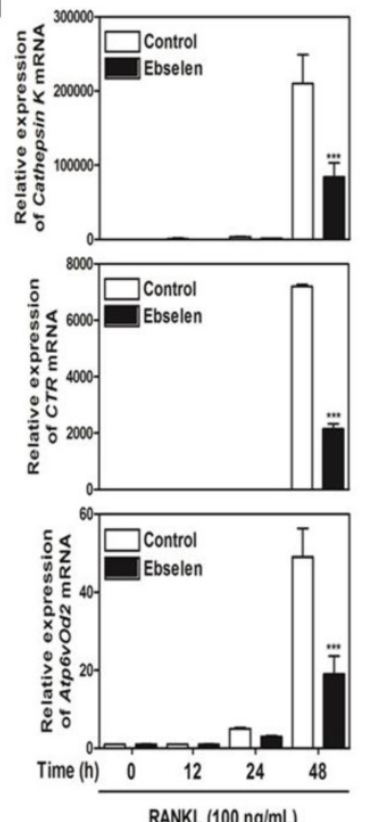

Fig. 4. Ebselen decreases actin ring formation and the bone resorption activity of mature osteoclasts. (A) BMMs were cultured with $M-C S F(30 \mathrm{ng} / \mathrm{mL})$ and $\mathrm{RANKL}(100 \mathrm{ng} / \mathrm{mL})$ and were posttreated with the indicated concentrations of ebselen. The cells were fixed, permeabilized, and stained with phalloidin and DAPI and were examined under a confocal laser-scanning microscope. (B) The number of AR+ osteoclasts and AR- osteoclasts were counted and compared with the total number of osteoclasts that contain three or more nuclei. The graph exhibits the comparison between relative percentage of osteoclasts that express normal actin ring (AR+) and disrupted actin ring (AR-). ${ }^{* * *} P<0.001$ and ${ }^{* *} P<0.01$ versus $A R+$ osteoclasts in control group; \#\#P $<0.001$ and \#P $\quad 0.01$ versus AR- osteoclasts in control group. (C) BMMs were pretreated with or without ebselen $(10 \mu \mathrm{M})$ for $1 \mathrm{~h}$ in the presence of $\mathrm{M}-\mathrm{CSF}(30 \mathrm{ng} / \mathrm{mL})$ and were stimulated with RANKL $(100 \mathrm{ng} / \mathrm{mL})$ for the indicated time. The mRNA expression of genes encoding OC- and DC-STAMP was analyzed by performing real-time RT-PCR; $n=3$, **** $P<0.001$ and ${ }^{* *} P<0.01$ versus control group. (D) Mature osteoclasts from the co-culture system were seeded in a 48-well plate for $6 \mathrm{~h}$, in a hydroxyapatite-coated plate for $24 \mathrm{~h}$, or in dentin slices for $48 \mathrm{~h}$ with or without ebselen. Cells attached to the 48 -well plate were stained with TRAP solution and those attached to the hydroxyapatite-coated plate were removed, and the plates were photographed under a light microscope. (E) The number of TRAP-positive MNCs (nuclei, $>3$ ) was determined. (F) Resorption pit areas in the hydroxyapatite-coated plate were quantified using Image-Pro Plus (Ver. 4.5 ) software; $\mathrm{n}=3$, ${ }_{* * * *} P<0.001$ versus control group. (G) Resorption pit areas in dentin slices were quantified using Image-Pro Plus $($ Ver. 4.5$)$ software; $n=3$, $* P<0.05$ versus control group. $(H)$ The mRNA expression of genes encoding cathepsin K, CTR, and Atp6vOd2 was analyzed by performing real-time RT-PCR; $n=3$, **** $P<0.001$ versus control group.

\section{Ebselen restores LPS-induced inflammatory bone loss in vivo}

To confirm whether the in vitro effects of ebselen on osteoclast formation and functions were also observed in vivo, we examined the effects of ebselen in a mouse model of LPS-induced inflammatory bone erosion. Mice were intraperitoneally injected with LPS and were treated with ebselen or PBS. After 8 days, the left femurs of sacrificed mice were analyzed by performing $\mu-\mathrm{CT}$ and the right femurs were stained with H\&E. LPS-induced reduction in bone mass was observed in the femurs of PBS-treated mice compared with that in the femurs of control mice. However, 3D visualization showed partial recovery of bone volume in mice treated with LPS and ebselen (Fig. 5A). Morphometric analysis of the femurs of mice treated with only LPS showed decreased level of BV/TV and increased level of Tb.Sp while those of mice treated with LPS and ebselen showed restoration of BV/TV, Tb.Sp, and Tb.Th (Fig. 5B). Histological analysis confirmed that ebselen treatment recovered LPS-induced loss of the trabecular bone matrix and suppressed TRAP-positive osteoclast formation in vivo (Figs. 5C, D). These results indicated that ebselen exerted inhibitory effects on osteoclast formation and subsequent bone resorption in vivo.

\section{Ebselen exerts anti-osteoclastic activity via targeting NMDA receptor.}

Lastly, we figured out the potential target protein of ebselen to regulate osteoclast differentiation and function. As shown in Fig. 6A and B, NMDA partially recovered the inhibitory effect of ebselen on 
RANKL-mediated osteoclast formation. Also, the down-regulation of IKB, PI3K and Akt phosphorylation was reversed in the treatment of NMDA (Fig. 6C). These results suggested that ebselen suppressed RANKL-induced osteoclastogenesis through interacting with NMDA.

\section{Discussion}

To date, most medications for treating osteoporosis target osteoclastic bone resorption (antiresorptive effects) and/or osteoblastic bone formation (anabolic effects). However, bisphosphonate, an antiresorptive agent, increases the risk of osteonecrosis of the jaw in osteoporotic patients [23]. In addition, parathyroid hormone, an anabolic skeletal agent, exerts several side effects, including hypercalcemia with renal failure, tumors, and nausea [24, 25]. To overcome these limitations and to identify a novel antiosteoporotic agent, we examined the therapeutic efficacy of the seleno-organic compound ebselen, a promising antioxidant with clinical safety and few side effects, by determining its effects on osteoclast differentiation and function both in vitro and in vivo.

RANKL and OPG secreted by preosteoblasts/stromal cells are required for initiating osteoclast differentiation. RANKL, a type II homotrimeric transmembrane protein, stimulates the release of

A
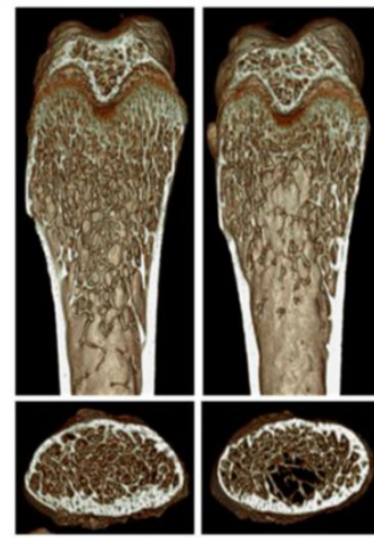

C

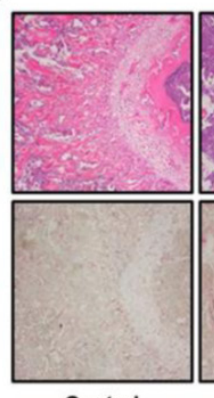

Control

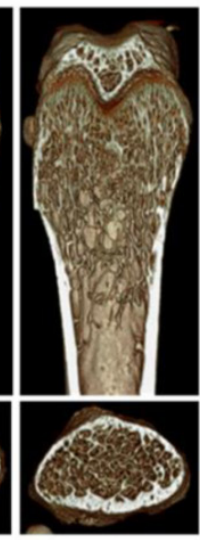

Ebselen

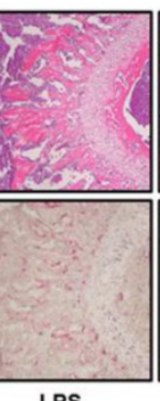

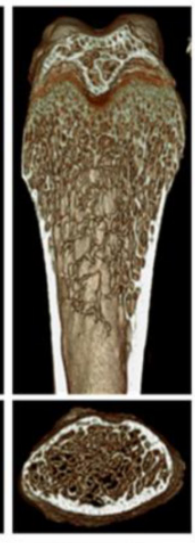
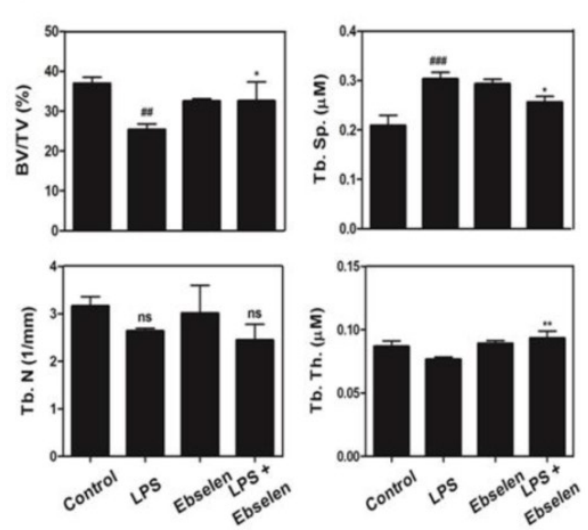

LPS+Ebselen

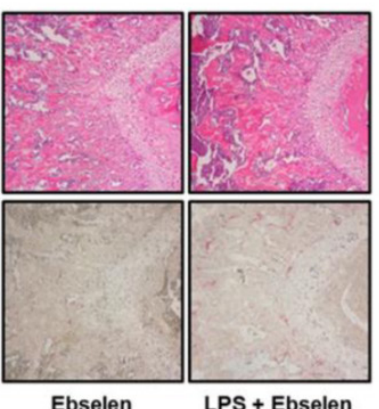

H\&E

D

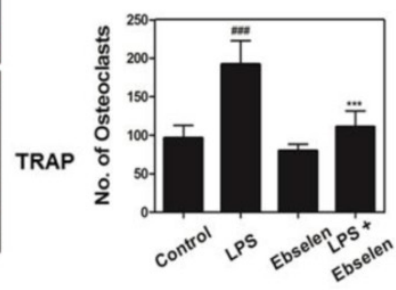

Fig. 5. Ebselen alleviates LPS-induced bone loss in a mouse model. (A) Mice were sacrificed on day 8 after the first LPS injection, and radiographs of the longitudinal and transverse sections of the proximal femurs were obtained using a $\mu$-CT scanner. (B) BV/TV, Tb.Sp, Tb.Th, and Tb.N of the femurs were determined by analyzing the $\mu$-CT data

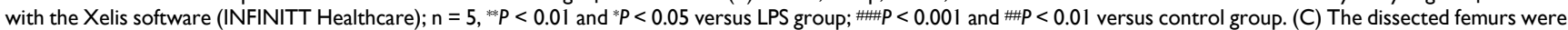
fixed, decalcified, embedded, and sectioned. The sections were stained with TRAP (bottom) and H\&E (top). (D) The number of osteoclasts per field of tissue was measured by

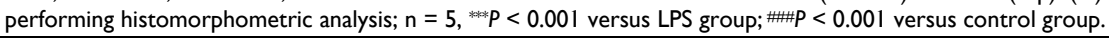


A

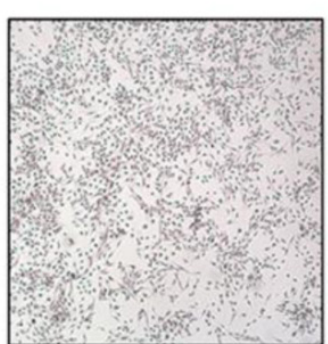

M-CSF

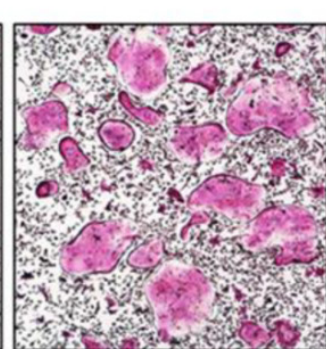

M-CSF + RANKL
B

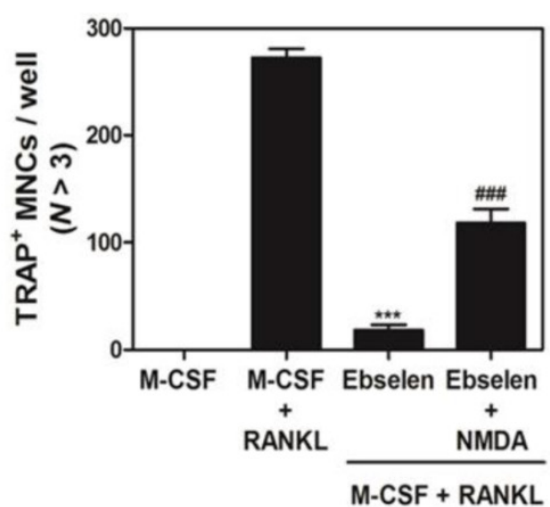

M-CSF + RANKL
M-CSF + RANKL

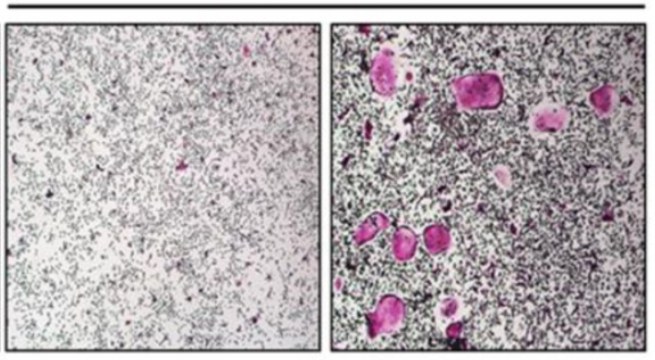

Ebselen
Ebselen + NMDA
C

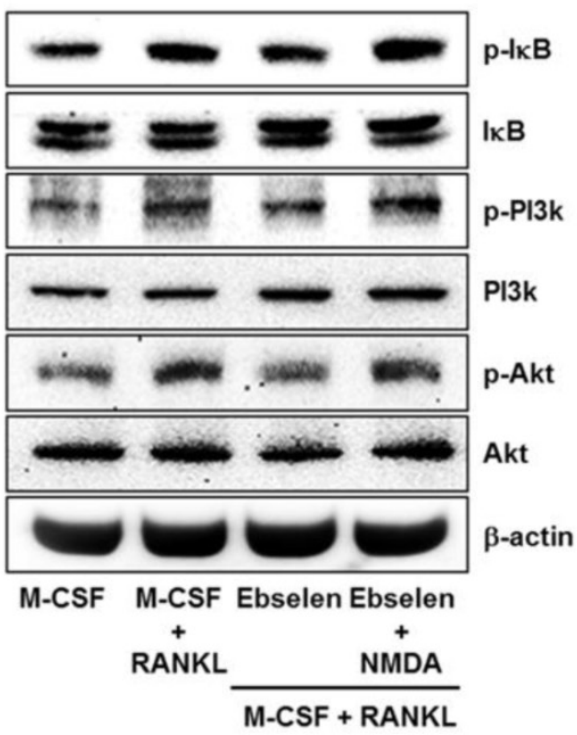

Fig. 6. NMDA agonist reverses the inhibitory effects of ebselen on osteoclast differentiation. (A) BMMs were cultured in the 4 different groups for 4 days including, only $M$-CSF $(30 \mathrm{ng} / \mathrm{mL}), M-C S F(30 \mathrm{ng} / \mathrm{mL})+$ RANKL $(100 \mathrm{ng} / \mathrm{mL})$, M-CSF $(30 \mathrm{ng} / \mathrm{mL})+$ RANKL $(100 \mathrm{ng} / \mathrm{mL})+$ ebselen, or M-CSF $(30 \mathrm{ng} / \mathrm{mL})+\mathrm{RANKL}(100 \mathrm{ng} / \mathrm{mL})+$ ebselen + NMDA $(100$ $\mu M)$. The cells were fixed, permeabilized, and stained with TRAP solution. TRAP-positive MNCs were photographed under a light microscope. (B) The number of TRAP-positive MNCs (nuclei $>3$ ) was determined; $n=3$, ${ }^{* *} P<0.001$ versus $M-C S F+R A N K L$ group and $\$ P<0.001$ versus M-CSF $+R A N K L+$ ebselen group. (C) BMMs were pretreated with or without ebselen or both ebselen and NMDA $(100 \mu \mathrm{M})$ for $1 \mathrm{~h}$ in the presence of M-CSF $(30 \mathrm{ng} / \mathrm{mL})$ before RANKL $(100 \mathrm{ng} / \mathrm{mL})$ stimulation for $15 \mathrm{~min}$. Whole-cell lysates were analyzed by performing western blotting with the indicated antibodies. $\beta$-actin was used as an internal control.

Osteoclast precursor-induced binding of osteoblast-derived RANKL to RANK activates several early signal transducers, including IKB, PI3K/Akt, p38, ERK, and JNK, in the early stage of osteoclastogenesis. In these signal pathways, PI3K/Akt signaling cascade regulates expression of NFATc1 during osteoclastogenesis. The cells with overexpression of Akt significantly induced translocation of NFATc1 in the nucleus of osteoclasts rather than cytoplasmic region. The NF- $\kappa$ B protein bound to the I $\kappa$ B has been known to induce early stage of osteoclast differentiation via c-Fos and subsequent activation of NFATc1. Also, the activation of c-Fos is strongly inhibited in NF- $\kappa$ B knockout mice cells [31-35]. As shown in Fig. 2, we observed that ebselen negatively regulated the expression of c-Fos and NFATc1 through interrupting RANKL-induced PI3K, Akt and IkB phosphorylation (Fig. 2). A study identified the role of the Akt/NF-kB pathway in osteoclast survival. Akt inhibition or NF- $\mathrm{KB}$ activation accelerates the apoptosis of osteo- clasts derived from the bone marrow, a co-culture with stromal cells, and the spleen [36]. Additionally, in mitochondrial pathway of caspase-dependent apoptosis, active expression of Akt promoted inactivation of well-defined pro-apoptotic proteins, caspase-3 and -9 via translocation of NF- $\mathrm{KB}$ to the nucleus, resulting in increased resistance to TNF-a-mediated apoptosis [37]. Therefore, we investigated whether ebselen regulated osteoclast apoptosis. We observed that post-treatment with ebselen on day 3 inhibited the formation of TRAP-positive MNCs during RANKL-induced osteoclast differentiation by inducing the cleavage (activation) of caspase- 3 and caspase-9 (Fig. 3A, B, and C). These results indicated that ebselen negatively controlled the formation and survival of osteoclasts by targeting the Akt/NF-кB pathway and by inducing caspase-dependent apoptosis.

Next, we determined the effects of ebselen on actin cytoskeletal organization required for osteoclast 
function and on osteoclastic bone resorption in the late stage of osteoclast maturation. Adhesion of mature osteoclasts to the surface of the bone or a bone-like biomineral substrate induces the formation of a dense F-actin ring structure (also known as sealing zone) through a tight network of podosome cores that are interconnected by actin fibers. This indicates that bone resorption, which is the unique function of osteoclasts, is induced by the generation of the sealing zone through the linkage of podosomes [38]. Osteoclasts establish an acidic microenvironment between the bone and themselves. This microenvironment contains large amounts of hydrogen ions that are secreted by the action of vacuolar $\mathrm{H}(+)$-ATPase-type proton pump located in the ruffled border and results in the dissolution of the bone matrix $[39,40]$. As expected, we observed that ebselen disrupted the organization of F-actin in cytoskeletal structures and significantly reduced the percentage of actin ring-positive osteoclasts in a dose-dependent manner and decreased the mRNA expression of osteoclast fusion-related genes encoding OC- and DC-STAMP (Figs. 4A, B, and C). Furthermore, ebselen directly inhibited bone resorption without exerting any cytotoxic effects on mature osteoclasts in the co-culture system and downregulated the mRNA expression of genes encoding cathepsin K, CTR, and Atp6vOd2, which are essential for osteoclastic bone degradation (Fig. 4D, E, F, G, and H). These results suggested that ebselen negatively regulated $\mathrm{F}$-actin ring formation and subsequent bone resorption in the late stage of osteoclast maturation.

Based on these in vitro effects of ebselen on osteoclast differentiation and function, we evaluated the effect of ebselen on inflammation-induced bone loss in mice by performing $\mu-\mathrm{CT}$ and histological analyses. LPS-induced acute systemic inflammation decreases bone mass by inducing host-derived inflammatory cytokines and by stimulating osteoclast formation and function both in vitro and in vivo. Mice that are intraperitoneally injected with LPS show abnormally increased erosion of bone surfaces [41-43]. We observed that ebselen significantly recovered bone loss and various bone-related $\mu$-CT parameters in LPS-treated mice (Fig. 5). In addition, ebselen inhibited the degradation of the trabecular bone matrix and eliminated TRAP-positive osteoclasts in the bone tissue. Although slight reduction of $\mathrm{BV} / \mathrm{TV}$ in only ebselen-treated mice compared with control group possibly due to the weaker inhibitory effect of ebselen on osteoblast differentiation was detected (Fig. S1), we proved the significant therapeutic effect of ebselen in inflammatory bone erosion condition.

Finally, we tried to disclose the potential target of ebselen to regulate osteoclast differentiation and activation in bone metabolism. Previously, it was proved that ebselen exerts neuroprotective effects by interacting with redox-sensitive N-methyl-Daspartate (NMDA) receptor [44]. In bone metabolism, the expression of this glutamate receptor is induced by osteoclast precursors, including RAW 264.7 cells and mouse bone marrow cells and is capable of promoting RANKL-mediated osteoclast differentiation [45]. These facts allowed us to hypothesize that ebselen has anti-osteoclastogenic effects via targeting NMDA receptor. Thus, we performed TRAP staining and western blotting using NMDA receptor agonist, NMDA to reveal that whether NMDA receptor is a potential molecular target of ebselen. As a result, ebselen significantly suppressed the formation of TRAP-positive osteoclasts induced by RANKL and this inhibitory effect of ebselen was recovered in the condition of NMDA treatment. Also, NMDA recovered the inhibitory effects of ebselen on RANKL-dependent phosphorylation of IкB, PI3K, and Akt in early signaling pathways. These results suggest that ebselen shows anti-osteoclastogenic effects via NMDA receptor.

Together, these results indicated that ebselen interfered with the RANKL-induced Akt/IkB signaling pathway via NMDA receptor, thus activating caspase-dependent apoptosis during osteoclastogenesis. In addition, ebselen inhibited the formation of the F-actin structure and subsequent bone resorption. These anti-osteoclastogenic effects were also observed in the mouse model of LPS-induced bone loss, suggesting that ebselen should be considered for treating metabolic bone diseases such as osteoporosis. Although we focused the in vitro and in vivo effects of ebselen on osteoclast differentiation and function in this manuscript, further studies are needed to evaluate relationship between ebselen and bone remodeling.

\section{Supplementary Material}

Figure S1. http://www.ijbs.com/v12p0478s1.pdf

\section{Acknowledgments}

This study was supported by a grant from the Korean Health Technology R\&D Project, Ministry of Health \& Welfare, Republic of Korea (HI12C0110).

\section{Competing Interests}

The authors have declared that no competing interest exists.

\section{References}

1. Tung S, Iqbal J. Evolution, aging, and osteoporosis. Ann N Y Acad Sci. 2007; 1116: 499-506.

2. Ginaldi L, Di Benedetto MC, De Martinis M. Osteoporosis, inflammation and ageing. Immun Ageing. 2005; 2: 14. 
3. Bruunsgaard H. Effects of tumor necrosis factor-alpha and interleukin-6 in elderly populations. Eur Cytokine Netw. 2002; 13: 389-91.

4. Müller B. Cytokine imbalance in non-immunological chronic disease. Cytokine. 2002; 18: 334-9.

5. Kim JH, Jin HM, Kim K, et al. The mechanism of osteoclast differentiation induced by IL-1. J Immunol. 2009; 183: 1862-70.

6. Manolagas SC. From estrogen-centric to aging and oxidative stress: a revised perspective of the pathogenesis of osteoporosis. Endocr Rev. 2010; 31: 266-300.

7. Lee NK, Choi YG, Baik JY, et al. A crucial role for reactive oxygen species in RANKL-induced osteoclast differentiation. Blood. 2005; 106: 852-9.

8. Lander HM. An essential role for free radicals and derived species in signal transduction. FASEB J. 1997; 11: 118-24.

9. Rhee SG. Redox signaling: hydrogen peroxide as intracellular messenger. Exp Mol Med. 1999; 31: 53-9.

10. Garrett IR, Boyce BF, Oreffo RO, et al. Oxygen-derived free radicals stimulate osteoclastic bone resorption in rodent bone in vitro and in vivo. J Clin Invest. 1990; 85: 632-9.

11. Müller A, Cadenas E, Graf P, et al. A novel biologically active seleno-organic compound-I. Glutathione peroxidase-like activity in vitro and antioxidant capacity of PZ 51 (ebselen). Biochem Pharmacol. 1984; 33: 3235-9.

12. Wendel A, Fausel $M$, Safayhi $H$, et al. A novel biologically active seleno-organic compound-II. Activity of PZ 51 in relation to glutathione peroxidase. Biochem Pharmacol. 1984; 33: 3241-5.

13. Saluk J, Bijak M, Nowak $\mathrm{P}$, et al. Evaluating the antioxidative activity of diselenide containing compounds in human blood. Bioorg Chem. 2013; 50: 26-33.

14. Tewari R, Sharma V, Koul N, et al. Ebselen abrogates TNFalpha induced pro-inflammatory response in glioblastoma. Mol Oncol. 2009; 3: 77-83.

15. Yoshizumi M, Kogame T, Suzaki $Y$, et al. Ebselen attenuates oxidative stress-induced apoptosis via the inhibition of the c-Jun N-terminal kinase and activator protein-1 signaling pathway in PC12 cells. Br J Pharmacol. 2002; 136: 1023-32.

16. Hanavan PD, Borges CR, Katchman BA, et al. Ebselen inhibits QSOX1 enzymatic activity and suppresses invasion of pancreatic and renal cancer cell lines. Oncotarget. 2015; 6: 18418-28.

17. Mukherjee S, Weiner WS, Schroeder CE, et al. Ebselen inhibits hepatitis C virus NS3 helicase binding to nucleic acid and prevents viral replication. ACS Chem Biol. 2014; 9: 2393-403.

18. Chew P, Yuen DY, Koh P, et al. Site-specific antiatherogenic effect of the antioxidant ebselen in the diabetic apolipoprotein E-deficient mouse. Arterioscler Thromb Vasc Biol. 2009; 29: 823-30.

19. Singh N, Halliday AC, Thomas JM, et al. A safe lithium mimetic for bipolar disorder. Nat Commun. 2013; 4: 1332

20. Kim JY, Cheon YH, Oh HM, et al. Oleanolic acid acetate inhibits osteoclast differentiation by downregulating PLC $2-\mathrm{Ca}(2+)-N F A T c 1$ signaling, suppresses bone loss in mice. Bone. 2014; 60: 104-11.

21. Baek JM, Kim JY, Cheon YH, et al. Dual effect of chrysanthemum indicum extract to stimulate osteoblast differentiation and inhibit osteoclast formation and resorption in vitro. Evid Based Complement Alternat Med. 2014; 2014: 176049.

22. Lakkakorpi PT, Väänänen HK. Kinetics of the osteoclast cytoskeleton during the resorption cycle in vitro. J Bone Miner Res. 1991; 817-26.

23. Khosla S, Burr D, Cauley J, et al. Bisphosphonate-associated osteonecrosis of the jaw: report of a task force of the American Society for Bone and Mineral Research. J Bone Miner Res. 2007; 22: 1479-91.

24. Neer RM, Arnaud CD, Zanchetta JR, et al. Effect of parathyroid hormone (1-34) on fractures and bone mineral density in postmenopausal women with osteoporosis. N Engl J Med. 2001; 344: 1434-41.

25. Ponnapakkam T, Katikaneni R, Sakon J, et al. Treating osteoporosis by targeting parathyroid hormone to bone. Drug Discov Today. 2014; 19: 204-8.

26. Boyce BF, Xing L. Biology of RANK, RANKL, and osteoprotegerin. Arthritis Res Ther. 2007; 9 Suppl 1: S1.

27. Kong YY, Yoshida H, Sarosi I, et al. OPGL is a key regulator of osteoclastogenesis, lymphocyte development and lymph node organogenesis. Nature. 1999; 397: 315-23.

28. Schneeweis LA, Willard D, Milla ME. Functional dissection of osteoprotegerin and its interaction with receptor activator of NF-kappaB ligand. J Biol Chem. 2005; 280: 41155-64.

29. Shalhoub V, Faust J, Boyle WJ, et al. Osteoprotegerin and osteoprotegerin ligand effects on osteoclast formation from human peripheral blood mononuclear cell precursors. J Cell Biochem. 1999; 72: 251-61.

30. Udagawa $\mathrm{N}$, Takahashi $\mathrm{N}$, Yasuda $\mathrm{H}$, et al. Osteoprotegerin produced by osteoblasts is an important regulator in osteoclast development and function. Endocrinology. 2000; 141: 3478-84.

31. Moon JB, Kim JH, Kim K, et al. Akt induces osteoclast differentiation through regulating the GSK3b/NFATc1 signaling cascade. J Immunol. 2012; 188: 163-9.

32. Yamashita T, Yao Z, Li F, et al. NF-kappaB p50 and p52 regulate receptor activator of NF-kappaB ligand (RANKL) and tumor necrosis factor-induced osteoclast precursor differentiation by activating c-Fos and NFATc1. J Bio Chem. 2007; 282: 18245-53.

33. Boyle WJ, Simonet WS, Lacey DL. Osteoclast differentiation and activation. Nature. 2003; 423: 337-42.

34. Grigoriadis AE, Wang ZQ, Cecchini MG, et al. c-Fos: a key regulator of osteoclast-macrophage lineage determination and bone remodeling. Science. 1994; 266: 443-8.
35. Takayanagi $\mathrm{H}$. The role of NFAT in osteoclast formation. Ann N Y Acad Sci. 2007; 1116: 227-37.

36. Gingery A, Bradley E, Shaw A, et al. Phosphatidylinositol 3-kinase coordinately activates the MEK/ERK and AKT/NFkappaB pathways to maintain osteoclast survival. J Cell Biochem. 2003; 89: 165-79.

37. Yi T, Lee HL, Cha HJ, et al. Epidermal growth factor receptor regulates osteoclast differentiation and survival through cross-talking with RANK signaling. J Cell Physiol. 2008; 217: 409-22.

38. Väänänen HK, Horton M. The osteoclast clear zone is a specialized cell-extracellular matrix adhesion structure. J Cell Sci. 1995; 108: 2729-32.

39. Teitelbaum SL. Bone resorption by osteoclasts. Science. 2000; 289: 1504-8.

40. Hakeda Y, Kumegawa M. Osteoclasts in bone metabolism. Kaibogaku Zasshi. 1991; 66: 215-25.

41. Ishihara $\mathrm{Y}$, Nishihara $\mathrm{T}$, Make E, et al. Role of interleukin-1 and prostaglandin in in vitro bone resorption induced by Actinobacillus actinomycetemcomitans lipopolysaccharide. J Periodontal Res. 1991; 26: 155-60.

42. Orcel P, Feuga M, Bielakoff J, et al. Local bone injections of LPS and M-CSF increase bone resorption by different pathways in vivo in rats. Am J Physiol. 1993; 264: E391-7.

43. Sakuma Y, Tanaka K, Suda M, et al. Impaired bone resorption by lipopolysaccharide in vivo in mice deficient in the prostaglandin E receptor EP4 subtype. Infect Immun. 2000; 68: 6819-25.

44. Herin GA, Du S, Aizenman E. The neuroprotective agent ebselen modifies NMDA receptor function via the redox modulatory site. J Neurochem. 2001; 78. 1307-14.

45. Merle B, Itzstein C, Delmas PD, et al. NMDA glutamate receptors are expressed by osteoclast precursors and involved in the regulation of osteoclastogenesis. J Cell Biochem. 2003; 90: 424-36. 